\title{
On the role of language membership information during word recognition in bilinguals: Evidence from flanker-language congruency effects
}

\author{
Mathieu Declerck $^{1,2} \cdot$ Joshua Snell ${ }^{1,2}$ - Jonathan Grainger ${ }^{1,2}$
}

Published online: 21 September 2017

(C) Psychonomic Society, Inc. 2017

\begin{abstract}
According to some bilingual language comprehension models (e.g., BIA), language membership information has a direct influence on word processing. However, this idea is not shared by all models (e.g., BIA+). To investigate this matter, we manipulated the language membership of irrelevant flanking words while French-English bilinguals performed a lexical decision task on centrally located target words and nonwords. The target words were either French or English words, flanked by words that were either in the same language as the target (language congruent) or in the other language (language incongruent). We found that lexical decisions to the target words were harder in the languageincongruent condition, indicating that language membership information was extracted from the flanking words and that this affected identification of the central target words, as predicted by the architecture of the BIA model.
\end{abstract}

Keywords Bilingualism $\cdot$ Language membership $\cdot$ Word recognition $\cdot$ Flanker task

To produce a word in a given language, bilinguals need to know which of their two languages should be used on that particular occasion. One means of achieving this is via language tags (Green, 1998) or language nodes (Grainger \&

Electronic supplementary material The online version of this article (https://doi.org/10.3758/s13423-017-1374-9) contains supplementary material, which is available to authorized users.

Mathieu Declerck

mathieu.declerck@univ-amu.fr

1 Laboratoire de Psychologie Cognitive, Aix-Marseille Université and Centre National de la Recherche Scientifique, Marseille, France

2 Centre National de la Recherche Scientifique, Paris, France
Dijkstra, 1992) that encode language membership information, such that the conjoint activation of a given language tag/node (e.g., "English") and a given concept (e.g., "domestic animal that barks") leads to activation of the appropriate phonological word form (/dog/) and preparation of the corresponding articulatory output. Although language membership information is a necessary prerequisite for language production, this is not the case for language comprehension in bilinguals, given that most words have language-specific phonological and orthographic representations, and that context can be used for disambiguation when this is not the case.

The role of language membership during bilingual language comprehension is captured in two seminal models, the bilingual interactive activation (BIA) model (Grainger \& Dijkstra, 1992), on the one hand, and the BIA+ model (Dijkstra \& van Heuven, 2002), on the other. According to the BIA model, language membership information influences language comprehension in bilinguals. More specifically, language nodes are automatically activated upon presentation of either a word in a given language or a nonlinguistic stimulus that is associated with a specific language, and language node activity modulates the relative activation level of the lexical representations in each language (see Grainger, Midgley, \& Holcomb, 2010, for a more detailed discussion).

On the other hand, the BIA+ model cannot capture such automatic influences of language membership on word recognition, because language nodes do not feed back information to lexical representations in this model. ${ }^{1}$ Language node activity can only influence performance via decision-level mechanisms in the BIA+ model. It is therefore not immediately obvious how the BIA+ model can capture effects of

\footnotetext{
${ }^{1}$ This follows the tradition of modularist theories of linguistic information processing, wherein evidence for top-down feedback is captured by complex decision-level mechanisms (e.g., Norris, McQueen, \& Cutler, 2000).
} 
language membership in tasks in which such information is irrelevant to the task being performed. The removal of feedback from language nodes to lexical representations in the $\mathrm{BIA}+$ was motivated by experiments using language-specific lexical decision with interlingual homographs that varied in their language-specific frequency (e.g., Dijkstra, Timmermans, \& Schriefers, 2000). The key finding was that homographs that have a low frequency in the target language and a high frequency in the nontarget language (an example English target word for a Dutch-English bilingual would be "list," which means "ruse" in Dutch) are often not recognized as being a word in the target language (Dijkstra et al., 2000), suggesting that language node activity was not used to recover the appropriate interpretation of these interlingual homographs (see Dijkstra \& van Heuven, 2002, for further discussion). However, this finding can also be captured by models that integrate feedback from language nodes to lexical representations, such as the BIA model, by assuming that the stronger activation of the high-frequency nontarget interpretation of the interlingual homograph (i.e., the language-specific associations between orthography, phonology, and semantics) provides sufficient input to the nontarget language node for it to dominate processing on those specific trials.

Moreover, there is evidence that language membership information is automatically activated during language comprehension in bilinguals and can have a direct influence on word recognition processes. A primary example of such automatic effects of language membership is language switch costs that have been found in experiments where language membership information is not necessary to performing the task: In a generalized lexical decision experiment (e.g., responding "word" if the letter string is a real word, independent of which language it belongs to), Grainger and Beauvillain (1987) found that performance was worse when a word from the other language had been the target on the preceding trial (a language switch), as compared with trials with no change in language (see also, e.g., Thomas \& Allport, 2000). Hence, the language membership on the preceding trial could affect word processing in the current trial, even when language membership is irrelevant to the task. Similar language switch costs have been observed with tasks other than generalized lexical decision, such as semantic categorization (e.g., Macizo, Bajo, \& Paolieri, 2012) and number categorization (e.g., Hirsch, Declerck, \& Koch, 2015; for a review, see Declerck \& Philipp, 2015).

More recently, research has shown that even nonlinguistic cues to language membership can affect language comprehension in bilinguals. For example, interlocutor identity has been found to have an influence, to the extent that such information generates expectations as to which language will be heard (Martin, Molnar, \& Carreiras, 2016; Molnar, Ibáñez-Molina, \& Carreiras, 2015). Most important, however, is the finding that nonlinguistic cues can influence language comprehension even when the cues are not informative with respect to either the process of comprehension or the specific task being performed. Grainger, Declerck, and Marzouki (2017) presented pictures of either the French or UK national flag, along with French and English words to be classified in a generalized lexical decision task. The flag stimuli predicted neither the language of the word nor the lexical decision response, yet a flag-language congruency effect was found, with lexical decision responses being facilitated in the presence of the flag associated with the language of the target word.

In the present study we sought further evidence for an influence of language membership information on language comprehension in bilinguals, as predicted by the BIA model. To do so, we built on prior evidence from the flanker paradigm that semantic and syntactic information can be derived in parallel from target and flanker words (Snell, Declerck, \& Grainger, 2017; Snell et al, 2017a; Snell et al, 2017b; Snell, Meeter, \& Grainger, 2017). The key evidence was obtained in a variant of the flanker paradigm derived from the flankingletters lexical decision task of Dare and Shillcock (2013). In this version of the flanker paradigm, the target and flankers are aligned horizontally, with the target on fixation and space separating the target and the flanker stimuli located on either side. The stimulus duration is brief enough (150-170 ms) to discourage eye movements to the flanking stimuli. In the Snell, Declerck, and Grainger study, French-English bilinguals were asked to semantically categorize English target words (natural vs. artificial object), while these were flanked by their noncognate French translation equivalent or by an unrelated French control word. The results showed that semantic decisions to central target words were facilitated by translation flankers, thus indicating that semantic information had been extracted from the parafoveal flanker words. In Snell, Meeter, and Grainger's study, participants had to categorize central target words as being an adjective or a noun, and the flanker words could be from the same syntactic category as the target word or from the opposite category. Syntactic category judgments were facilitated by flankers from the same category, thus indicating that syntactic information had been extracted from the parafoveal flanker words.

This prior research suggests that other kinds of high-level linguistic knowledge, such as language membership information, could be extracted from briefly presented parafoveal flanker words. To examine this possibility, we asked French-English bilinguals to indicate whether a centrally located string of letters was a word or a nonword (generalized lexical decision) while we manipulated the language congruency of semantically and orthographically unrelated flanking words. More specifically, every target was flanked by a word from the same language (language congruent; e.g., talk dice talk) or from the other language (language incongruent; e.g., loup dice loup). We predicted that language membership information would be extracted automatically from the flanker 
words and that this would influence lexical decisions to the central target words, even though language membership information was irrelevant for the task (i.e., generalized lexical decision). Such a finding would be compatible with the architecture of the BIA model, but not with the BIA+ model.

\section{Method}

\section{Participants}

A total of 24 French-speaking participants that spoke English as their second language (16 female, eight male; mean age = 20.7 years) took part in this experiment. Prior to the experiment, the participants filled in a questionnaire about their French and English proficiency and completed a French (Brysbaert, 2013) and an English (Lemhöfer \& Broersma, 2012) vocabulary test. The questionnaire consisted of questions about the participants' ages of acquisition of the two languages, the average percentages of current language use, and the levels of speaking and reading skills in French and English on a 7-point scale, with 1 being very bad and 7 being very good (see Table 1).

\section{Apparatus, materials, and task}

In the present study, French-English bilinguals were presented words and nonwords with OpenSesame (Mathôt, Schreij, $\&$ Theeuwes, 2012), using a $1,920 \times 1,080$ pixel, $150-\mathrm{Hz}$ computer monitor. The French-English bilinguals' task was to categorize the central target string of letters as a word in either language or as a nonword.

We retrieved 60 four-letter English target words (frequency: 5.37 Zipf) from the British Lexicon Project lexicon (Keuleers, Lacey, Rastle, \& Brysbaert, 2012), and 60 fourletter French target words $(5.65 \mathrm{Zipf})$ from the French Lexicon Project lexicon (Ferrand et al., 2010). All targets contained no diacritics and were not inflected forms.

Table 1 Overview of demographic information ( $S D$ in brackets)

\begin{tabular}{lll}
\hline & French & English \\
\hline Age of acquisition & $0.5(1.4)$ & $8.4(3.0)$ \\
Currently used & $70.8(22.1)$ & $29.2(22.1)$ \\
Speaking & $6.4(0.7)$ & $4.3(1.0)$ \\
Reading & $6.5(0.7)$ & $5.1(1.0)$ \\
LexTALE & $88.8(4.7)$ & $71.5(10.7)$ \\
\hline
\end{tabular}

The information consists of the average age of acquisition of each language and the average percentage of time the participants currently spoke each one. Furthermore, the average self-rated scores are given for speaking and reading the two languages, as are the average LexTALE scores for both languages.
We coupled each target with a semantically and orthographically unrelated four-letter English word and a fourletter French word, both of which would serve as flankers. Hence, each target was presented in both flanker conditions across participants: once with flankers in the same language being presented to the left and right of the target (the targets and flankers were separated by one character space on each side), and once with flankers in the other language. The average frequencies were kept equal between the English (5.45 Zipf) and French (5.56 Zipf) flankers.

In a similar manner, we retrieved 120 nonword targets $(60$ "English" nonwords and 60 "French" nonwords) from the respective pseudoword counterparts of the English and French lexicons. These nonword targets were also coupled with both "English" and "French" nonword flankers.

\section{Procedure}

Prior to the experiment, the instructions were presented both orally and visually, and participants were instructed to respond as rapidly and accurately as possible. Then the participants performed two experimental blocks, consisting of 120 trials each, one with French target words and the other with English target words. Half of the trials would consist of target words, whereas the other half would consist of target nonwords. The nonwords were always flanked by nonwords, whereas target words would be flanked by words from the same language (i.e., language congruent) on $50 \%$ of the trials, and by words from the other language (i.e., language incongruent) on the other trials. The order of the language-congruent, languageincongruent, and nonword trials was completely random. Each of these experimental blocks was preceded by a practice block of 12 trials.

Every trial started with two centrally positioned vertical fixation bars (see Fig. 1). After $600 \mathrm{~ms}$, the target appeared between these fixation bars, with flanking stimuli to its left and right sides (the targets and flankers were separated by one character space on each side). After $170 \mathrm{~ms}$ the target and flankers disappeared, and participants had a maximum of 2,000 ms to indicate whether or not they had recognized the target as being a word. This was done with a left or right button press ("w" or "!," respectively, on an azerty keyboard), with the right button always corresponding to "word." A green or a red dot was then shown briefly $(600 \mathrm{~ms})$ at the center of the screen, depending on whether the participant's response was correct or incorrect, respectively, and after this the next trial would start.

\section{Analysis}

For the reaction time (RT) analysis, errors were discarded, as were RTs that were above or below two standard deviations from the mean (per participant). Overall, this resulted in the exclusion of $14.2 \%$ of the RT data. 


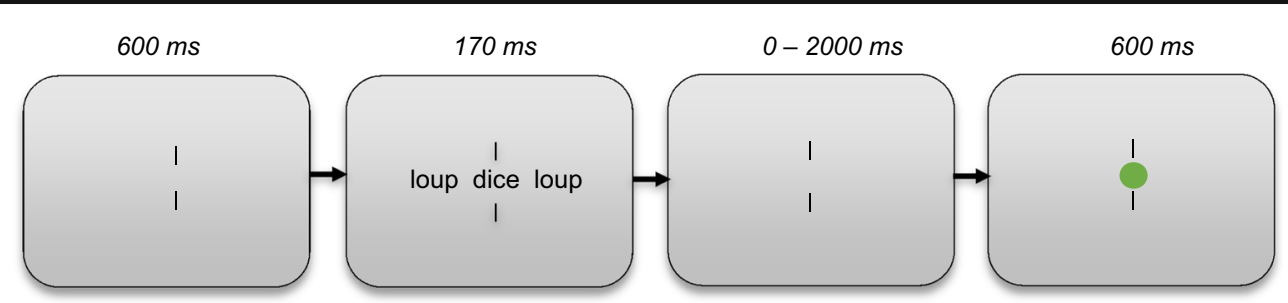

Fig. 1 Overview of the trial procedure. The size of the stimuli relative to the screen is exaggerated in this example

The RT data were analyzed using mixed-effects models (Baayen, Davidson, \& Bates, 2008), and the error data were analyzed using a logistic mixed model (Jaeger, 2008). Both participants and items were considered random factors, with all fixed effects (i.e., language [French vs. English], language congruency between target and flankers [congruent vs. incongruent], and their interaction) varying by all random factors (Barr, Levy, Scheepers, \& Tily, 2013). In the error analysis, the full random-effects model did not converge. This was resolved by using a model that contained intercepts for both participants and items but that only contained the byparticipant random slope for language. ${ }^{2}$

\section{Results}

As can be seen in Table 2, the RT data revealed a significant main effect of language, with English trials $(633 \mathrm{~ms})$ being slower than French trials (596 ms). Importantly, the main effect of language congruency was also significant, with incongruent language trials $(619 \mathrm{~ms})$ being slower than congruent trials $(608 \mathrm{~ms}) .{ }^{3}$ We found no significant interaction.

As can be seen in Table 3, only a significant main effect of language emerged in the error data, with a higher error rate in English trials $(14.9 \%)$ than in French trials $(6.8 \%$; see Table 4).

\section{Discussion}

In the present study, we investigated the role of language membership information during language comprehension in bilinguals. To this end, we implemented a bilingual flanker

\footnotetext{
${ }^{2}$ Comparing the fit of our reduced model (AIC: 1,705) with a full randomeffects model (AIC: 1,722) showed no significant difference $(p=.54)$. Thus, the observed effects were not due to variability that was not captured by the model.

${ }^{3}$ We replicated this effect with a new sample of 24 French-English bilinguals from the same participant pool. The setup of this experiment was identical to that of the main experiment, except that participants performed two additional mixed-language blocks (French and English), as well as the two pure-language blocks (French or English). The results showed a main effect of language congruency, $b=21.3, S E=9.6, t=2.2$, with slower responses in incongruent $(620 \mathrm{~ms})$ than in congruent language trials $(608 \mathrm{~ms})$. Language congruency did not interact with the type of block, $b=21.2, S E=12.9, t=1.6(17 \mathrm{~ms}$ congruency effect in pure-language blocks, and $23 \mathrm{~ms}$ in mixed-language blocks).
}

task in which the flanking words could be presented either in the same language as the target word (language congruent) or in the other language (language incongruent). The language of the flanking words was irrelevant to the task being performed (generalized lexical decision on the central targets), yet our results showed better performance when the language of the flanker and target words was congruent (for a replication of the data, see note 3 ).

This language congruency effect suggests that language membership information can directly influence word recognition in bilinguals. Language membership information would be automatically extracted from the irrelevant flanker words, and this information would either help or hinder the processing of the central target words, depending on whether or not the target and flankers were from the same language. The original BIA model implements a specific mechanism for the automatic activation of language membership information and for the influence of such activation on word recognition in bilinguals. The language nodes of the BIA model receive input from language-specific stimuli, and this activation is used to regulate the relative activation level of lexical representations in each language. Thus, when the flankers were French words, this would lead to activation of the French language node and the subsequent inhibition of all English words, making it harder to process an English target word in the incongruent condition. The English language node would of course be activated by the English target word, but the overall activation of both language nodes is what determines the level of inhibition directed at lexical representations in one or the other language.

According to the BIA+ model, on the other hand, activity in language nodes cannot directly affect word recognition. Hence, this model predicted no impact of language congruency between flanker words and the target word, a prediction that was contradicted by our findings. It could be argued,

Table $2 b, t$ values, and standard errors in the RT analyses

\begin{tabular}{llll}
\hline Effects & $b$ Value & Standard Error & $t$ Value \\
\hline Intercept & 461.0 & 14.1 & $32.6^{* * * *}$ \\
Congruency & 18.6 & 8.6 & $2.2^{*}$ \\
Language & 38.2 & 11.6 & $3.3^{* *}$ \\
Congruency $\times$ Language & 7.3 & 10.8 & 0.7 \\
\hline
\end{tabular}

${ }^{*} p<.05,{ }^{* *} p<.01,{ }^{* * *} p<.001$. 
Table $3 \quad b, z$ values, and standard errors in the error analyses

\begin{tabular}{llll}
\hline Effects & $b$ Value & Standard Error & $z$ Value \\
\hline Intercept & -2.5 & 0.3 & $9.1^{* * * *}$ \\
Congruency & 0.1 & 0.2 & 0.5 \\
Language & 0.9 & 0.4 & $2.4^{*}$ \\
Congruency $\times$ Language & 0.4 & 0.3 & 1.3 \\
\hline
\end{tabular}

${ }^{*} p<.05,{ }^{* *} p<.01,{ }^{* * *} p<.001$.

however, that our results might have been driven by flanker language congruency affecting executive control processes (i.e., task/decision system) in the BIA+ model, by changing the criterion used for making a lexical decision response. Exactly how this might occur is not at all obvious, and one finding from our laboratory speaks against any role for adjustments in decision criteria as a cause of flanker effects in general (see the supplementary materials). In a simple monolingual lexical decision flanker experiment, we found that flanker lexicality (word vs. pseudoword vs. nonword) did not significantly influence lexical decision responses to central target words. This suggests that the flanker paradigm is a good reflection of the linguistic information processing that occurs prior to the involvement of decision mechanisms.

As well as their implications for bilingual language comprehension, our results also speak to the hotly debated issue of serial versus parallel word processing during reading. The observation that the language of parafoveal flanker words has an influence on the processing of the foveal target word provides further evidence in favor of the idea that higher-order processing can occur for multiple words in parallel, at least to the extent that the representation of language membership is considered to be a high-level phenomenon. We of course acknowledge the possibility that language membership information can be activated via sublexical cues (e.g., Casaponsa, Carreiras, \& Duñabeitia, 2014; Van Kesteren, Dijkstra, \& de Smedt, 2012), but would argue that the same representation of language membership information, such as the language nodes in the BIA model, receives input from both sublexical and lexical activity associated with a given language, and

Table 4 Overall RTs (in milliseconds) and percentages of errors (SDs in parentheses) as a function of language congruency (congruent vs. incongruent), and language (French vs. English)

\begin{tabular}{lll}
\hline & Congruent & Incongruent \\
\hline RT & & \\
French & $591(65)$ & $600(73)$ \\
English & $627(64)$ & $639(72)$ \\
Error rates & & $7.6(7.0)$ \\
French & $6.0(5.6)$ & $14.6(10.6)$ \\
English & $15.3(8.8)$ & \\
\hline
\end{tabular}

therefore that the influence of such language membership information necessarily involves postlexical processing.

In sum, our results indicate that the language of unattended flanker words can influence the processing of central target words during bilingual language comprehension. This is in line with models of bilingual word recognition that assume that the mechanism used to represent language membership information is also used to regulate the relative activation of lexical representations in each language, as is the case in the BIA model.

Author note The authors thank Sheena Franceschi and Agnès Guerre-Genton for their help in conducting the experiments. This project received funding from the European Union's Horizon 2020 research and innovation program under Marie Sklodowska-Curie Grant Agreement No. 706128. This research was also supported by grants ANR-11-LABX-0036 (BLRI), ANR-16-CONV-0002 (ILCB), and ANR-11-IDEX0001-02 from the French National Research Council (ANR).

\section{References}

Bayen, R. H., Davidson, D. J., \& Bates, D. M. (2008). Mixed-effects modeling with crossed random effects for subjects and items. Journal of Memory and Language, 59, 390-412. doi:https://doi. org/10.1016/j.jml.2007.12.005

Barr, D. J., Levy, R., Scheepers, C., \& Tily, H. J. (2013). Random effects structure for confirmatory hypothesis testing: Keep it maximal. Journal of Memory and Language, 68, 255-278. doi:https://doi. org/10.1016/j.jml.2012.11.001

Brysbaert, M. (2013). Lextale_FR: A fast, free, and efficient test to measure language proficiency in French. Psychologica Belgica, 53, 23 37.

Casaponsa, A., Carreiras, M., \& Duñabeitia, J. A. (2014). Discriminating languages in bilingual contexts: The impact of orthographic markedness. Frontiers in Psychology, 5, 424. doi:https://doi.org/10.3389/ fpsyg.2014.00424

Dare, N., \& Shillcock, R. (2013). Serial and parallel processing in reading: Investigating the effects of parafoveal orthographic information on nonisolated word recognition. Quarterly Journal of Experimental Psychology, 66, 417-428.

Declerck, M., \& Philipp, A. M. (2015). A review of control processes and their locus in language switching. Psychonomic Bulletin \& Review, $22,1630-1645$.

Dijkstra, T., Timmermans, M., \& Schriefers, H. (2000). On being blinded by your other language: Effects of task demands on interlingual homograph recognition. Journal of Memory and Language, 42, 445-464.

Dijkstra, T., \& van Heuven, W. J. (2002). The architecture of the bilingual word recognition system: From identification to decision. Bilingualism: Language and Cognition, 5, 175-197.

Ferrand, L., New, B., Brysbaert, M., Keuleers, E., Bonin, P., Méot, A., . . Pallier, C. (2010). The French Lexicon Project: Lexical decision data for 38,840 French words and 38,840 pseudowords. Behavior Research Methods, 42, 488-496. doi:https://doi.org/10.3758/BRM. 42.2 .488 
Grainger, J., \& Beauvillain, C. (1987). Language blocking and lexical access in bilinguals. Quarterly Journal of Experimental Psychology, 39, 295-319.

Grainger, J., Declerck, M., \& Marzouki, Y. (2017). On national flags and language tags: Effects of flag-language congruency in bilingual word recognition. Acta Psychologica, 178, 12-17.

Grainger, J., \& Dijkstra, T. (1992). On the representation and use of language information in bilinguals. In R. J. Harris (Ed.), Cognitive processing in bilinguals (pp. 207-220). Amsterdam, The Netherlands: North Holland. doi:https://doi.org/10.1016/ S0166-4115(08)61496-X

Grainger, J., Midgley, K. J., \& Holcomb, P. J. (2010). Re-thinking the bilingual interactive-activation model from a developmental perspective (BIA-d). In M. Kail \& M. Hickman (Eds.), Language acquisition across linguistic and cognitive systems (pp. 267-284). Philadelphia, PA: Benjamins.

Green, D. W. (1998). Mental control of the bilingual lexico-semantic system. Bilingualism: Language and Cognition, 1, 67-81.

Hirsch, P., Declerck, M., \& Koch, I. (2015). Exploring the functional locus of language switching: Evidence from a PRP paradigm. Acta Psychologica, 116, 1-6.

Jaeger, T. F. (2008). Categorical data analysis: Away from ANOVAs (transformation or not) and towards logit mixed models. Journal of Memory and Language, 59, 434-446. doi:https://doi.org/10. 1016/j.jml.2007.11.007

Keuleers, E., Lacey, P., Rastle, K., \& Brysbaert, M. (2012). The British Lexicon Project: Lexical decision data for 28,730 monosyllabic and disyllabic English words. Behavior Research Methods, 44, 287 304. doi:https://doi.org/10.3758/s13428-011-0118-4

Lemhöfer, K., \& Broersma, M. (2012). Introducing LexTALE: A quick and valid Lexical Test for Advanced Learners of English. Behavior
Research Methods, 44, 325-343. doi:https://doi.org/10.3758/ s13428-011-0146-0

Macizo, P., Bajo, T., \& Paolieri, D. (2012). Language switching and language competition. Second Language Research, 28, 131-149.

Martin, C. D., Molnar, M., \& Carreiras, M. (2016). The proactive bilingual brain: Using interlocutor identity to generate predictions for language processing. Scientific Reports, 6, 26171.

Mathôt, S., Schreij, D., \& Theeuwes, J. (2012). OpenSesame: An opensource, graphical experiment builder for the social sciences. Behavior Research Methods, 44, 314-324. doi:https://doi.org/10. 3758/s13428-011-0168-7

Molnar, M., Ibáñez-Molina, A., \& Carreiras, M. (2015). Interlocutor identity affects language activation in bilinguals. Journal of Memory and Language, 81, 91-104.

Norris, D., McQueen, J. M., \& Cutler, A. (2000). Merging information in speech recognition: Feedback is never necessary. Behavioral and Brain Sciences, 23, 299-325, disc. 325-370.

Snell, J., Declerck, M., \& Grainger, J. (2017a). Parallel semantic processing in reading revisited: Effects of translation equivalents in bilingual readers. Manuscript submitted for publication.

Snell, J., Meeter, M., \& Grainger, J. (2017b). Evidence for simultaneous syntactic processing of multiple words during reading. PLOS ONE, 12, e0173720. doi:https://doi.org/10.1371/journal.pone.0173720

Thomas, M. S. C., \& Allport, A. (2000). Language switching costs in bilingual visual word recognition. Journal of Memory and Language, 43, 44-66.

Van Kesteren, R., Dijkstra, T., \& de Smedt, K. (2012). Markedness effects in Norwegian-English bilinguals: Task-dependent use of languagespecific letters and bigrams. Quarterly Journal of Experimental Psychology, 65, 2129-2154. doi:https://doi.org/10.1080/17470218. 2012.679946 